\title{
Interactive web analysis and presentation of computer- controlled scanning electron microscopy data
}

\author{
Peter Riisager, Nynke Keulen, Uffe Larsen, Roger K. McLimans, Christian Knudsen and \\ Jørgen Tulstrup
}

In the following we describe the result of the Titan Project, an interactive web application (Titan) developed at the Geological Survey of Denmark and Greenland (GEUS) together with DuPont Titanium Technologies. The main aim of $\mathrm{Ti}$ $\tan$ is to make computer-controlled scanning electron microscopy (CCSEM) data, generated at GEUS, available via the internet. In brief, CCSEM is a method automatically to detect particles with a scanning electron microscope (SEM), and based on computer-controlled imagery to measure the chemistry and grain morphology of each particle in a given sample (Knudsen et al. 2005; Bernstein et al. 2008); Keulen et al. 2008. Titan makes data available on-line so that the user can interact with the data sets and analyse them using a web browser. In addition to CCSEM data, Titan contains a global database of titanium deposits and various reports. The web application is customised, such that the functionality and amount of data available for a given user depend on the privileges of that user.

\section{Data}

A prerequisite for making Titan available on the internet is that the data are stored in a relational database allowing for fast querying and retrieval. A detailed description of the data model is not given here, but we will make a short overview of the different data types and how the data are inserted and managed in the database. The data are stored in an Oracle $10 \mathrm{~g}$ database that is housed and maintained by GEUS (Tulstrup 2004).

\section{CCSEM data}

CCSEM data are uploaded to the Jupiter database via a desktop program developed with the Delphi software tool at GEUS (Fig. 1). Typical CCSEM samples consist of roughly one thousand grains. For each grain c. 30 analytical parameters are measured including elemental composition, grain size and grain shape. All parameters are stored in the database including the energy dispersive X-ray spectrum for each grain (from which the elemental composition of the grain is determined) as well as backscatter-mode SEM images of the sample. The number of measurements currently stored in the database exceeds 54 million (Fig. 2), and the number continues to grow at a rate of $c .15$ million per year. Once data are uploaded, each grain is classified according to a mineral classification scheme that is implemented using Oracle database PL/SQL procedures. The mineral classification scheme is based on the measured concentration of elements, and different schemes are available depending on the type of sample. For example, there is a mineral classification scheme for heavy mineral concentrates, while another scheme is applied for soil samples.

\section{Titanium deposits and reports}

Data on titanium deposits can be entered directly into the database through Titan, and at present it contains detailed information about more than 600 deposits around the world.

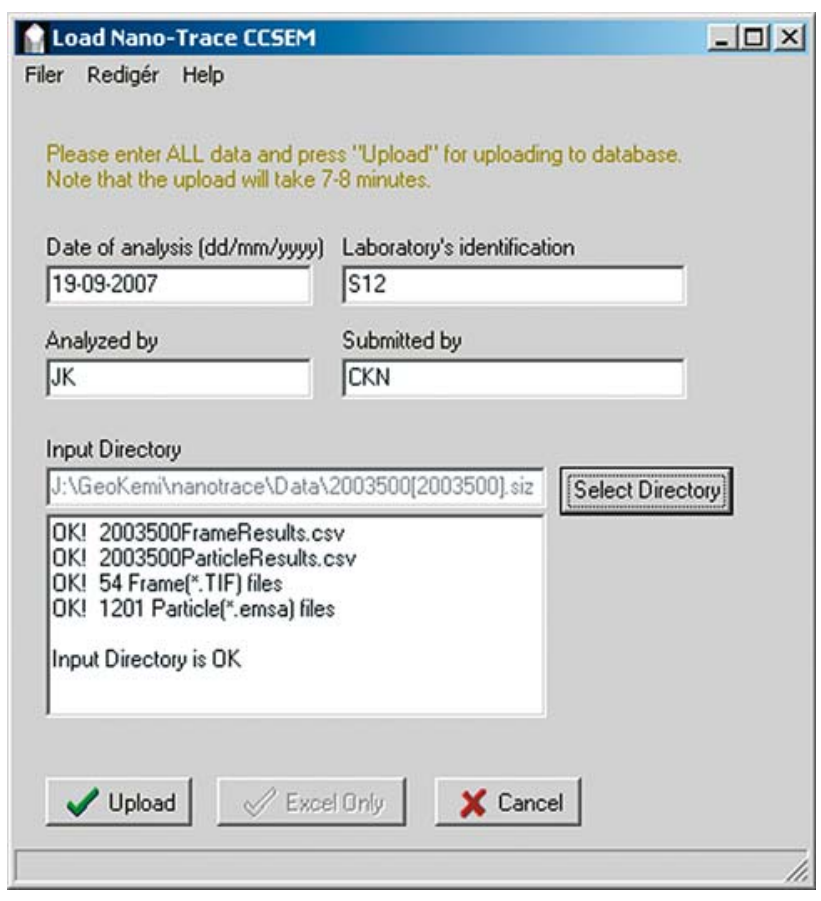

Fig. 1. Entry of sample metadata and uploading of CCSEM data to the database using a desktop programme. The energy dispersive X-ray spectrum for each grain and backscatter-mode SEM images of the sample are also uploaded. 


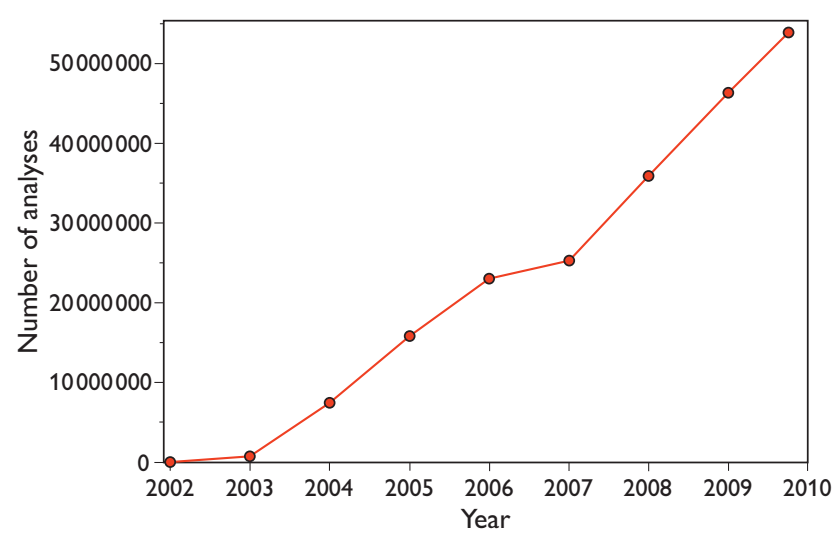

Fig. 2. Number of measurements in the Titan database as a function of time. Currently the database contains $c .54$ million measurements.

Reports and report metadata are uploaded to the database via the web application. The option to create, read, update, and delete deposits and reports is restricted to certain users and managed by strict user control.

\section{Web interface}

The web application is based on open-source software, mainly Java, and is running in an open-source JBoss Application Server housed and maintained by GEUS. Access to Titan requires login with username and password. Once logged in, the system assigns the user a specific role with specific priviliges allowing certain analytical functions. The user control is also extended to the individual CCSEM samples so that each group of users can see and interact with only a restricted subset of CCSEM samples. The CCSEM data can be accessed using an interactive geological map web page (Fig. 3) or by alphanumeric search criteria. When a sample is found, various recalculated data for that sample are available in table formats, and back-scatter mode SEM images or other files, such as outcrop pictures related to the sample, can be viewed or downloaded. If users want to work with the data off-line, data can be exported to Microsoft Excel format or a customised PDF report can be generated. Most importantly, the user has the option to visualise and interact with the data using various plots. For example, the elemental composition of all the mineral grains in a given sample can be presented in a scatter plot (Fig. 4), where the user defines the elements assigned to the $\mathrm{x}$ and $\mathrm{y}$ axes and which minerals should be plotted. The user can select which minerals to be plotted for analysis and comparison of apparent grain sizes (Fig. 5). This gives the user quick and easy access to make the desired analyses and plots. The on-line access has the added benefit that users around the world always have live access to the database, meaning that as soon as new data are uploaded the users can start interacting with them.

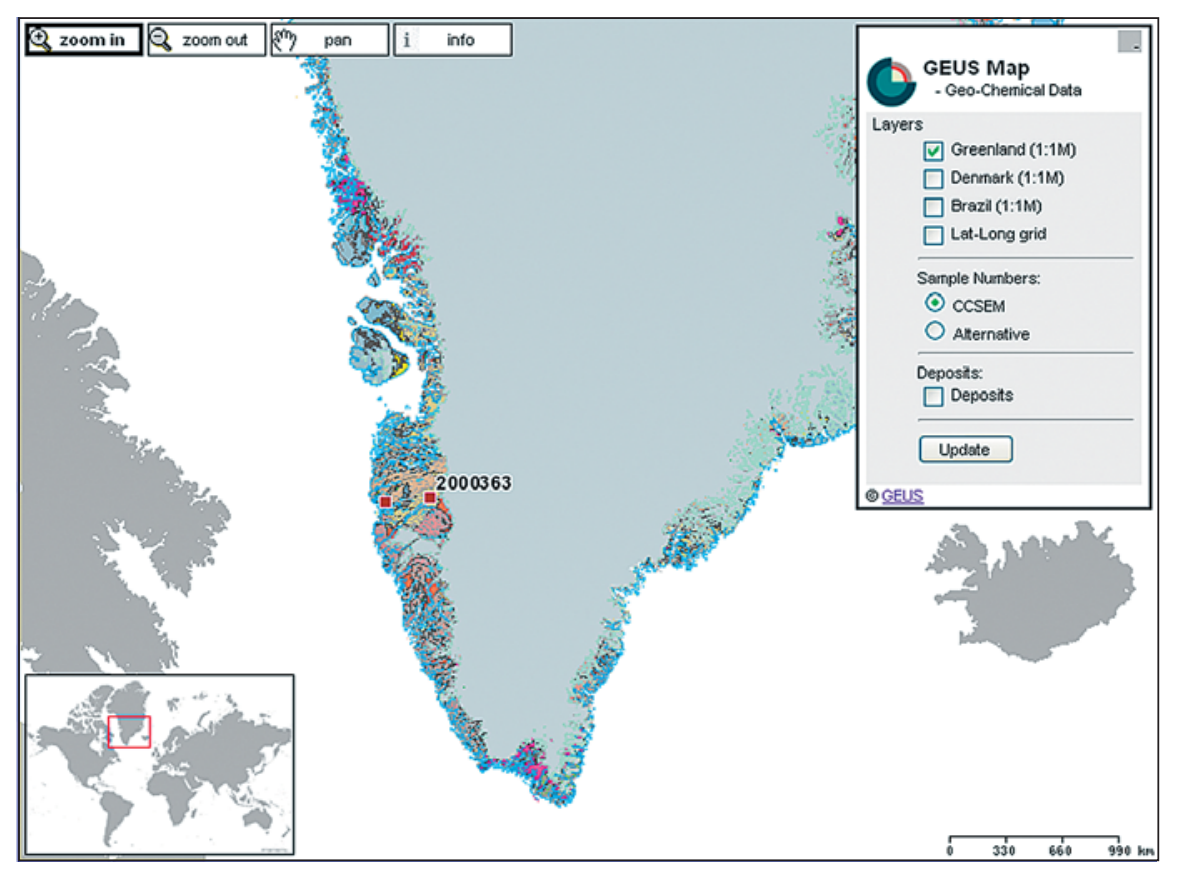

Fig. 3. The Titan web application includes an interactive map that allows the user to search for CCSEM samples based on their geographic sampling coordinates. Clicking on a sample on the map takes the user to other screen images where one can interact with the actual data (Figs 4, 5). 
Fig. 4. Analysis of CCSEM data with an interactive scatter plot using Titan. The user defines the elements assigned to the $\mathrm{x}$ and $\mathrm{y}$ axes, and which minerals are plotted.

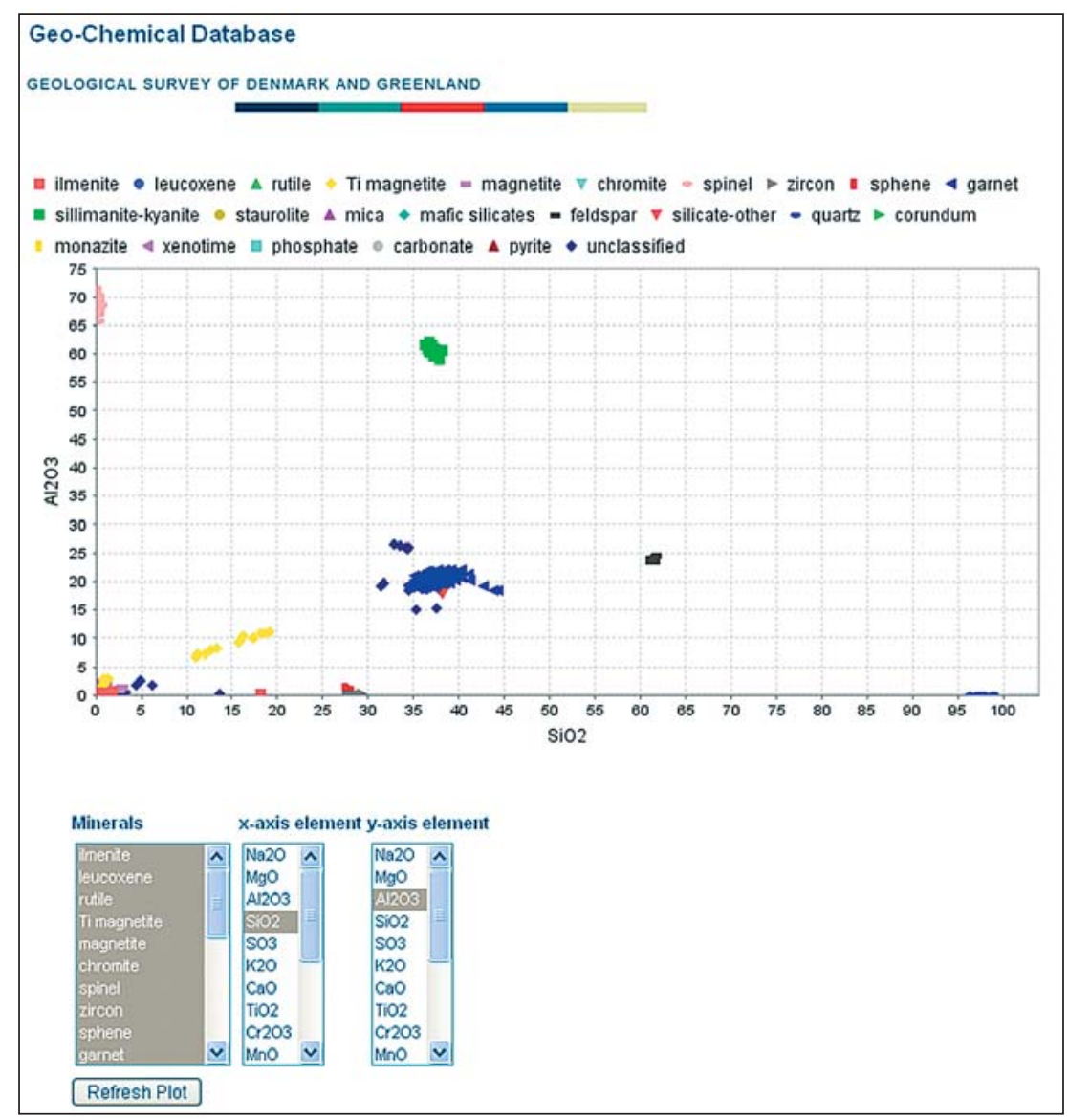

\section{Examples of applications}

Titan has successfully delivered data to scientists working at GEUS as well as various external end-users. In the following we will give some short examples of the use of Titan.

\section{Tracing kimberlite-indicator minerals for diamond prospection}

The elemental compositions of megacrystal and xenocrystal phases within kimberlitic rocks are used as an important diamond exploration tool. Preliminary studies have demonstrated the excellent potential of CCSEM to determine the elemental composition of minor elements in these minerals in a reliable and more cost-efficient manner than conventional electron microprobe analysis (Keulen et al. 2009).

\section{Exploration and ore deposit evaluation}

Mineralogical characterisation of sediments is a prerequisite for exploration and exploitation of valuable sediment occur- rences, such as heavy mineral deposits. With Titan we have characterised individual mineral particles in heavy mineral sands, with the aim of detecting heavy mineral ore deposits (Knudsen et al. 2005; Bernstein et al. 2008).

\section{Soil sample mineralogy for the cement industry}

Cement manufacture causes emission of large quantities of airborne pollutants including greenhouse gases. With Titan we have studied the raw materials used in cement production to optimise the performance of the grinding mill and the sintering process, with the ultimate aim to lower energy consumption at high temperatures (Keulen et al. 2008).

\section{Sediment provenance studies for oil exploration and sedimentary basin analysis}

Sediment provenance studies are an important aspect in the evaluation of possible sandstone reservoirs. Titan has served as an indispensable tool to determine source, compositional 


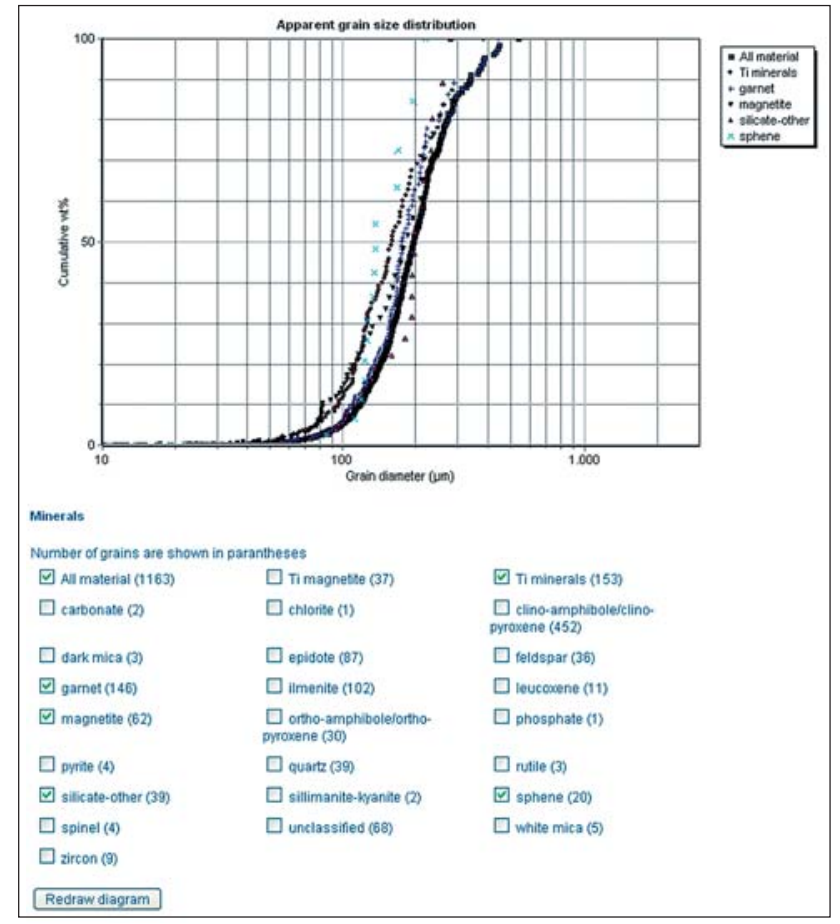

Fig. 5. Analysis and comparison of the apparent grain size distributions of the various mineral fractions in the sample using Titan. The plot is interactive, allowing the user to choose which minerals are plotted.

variation and sedimentary pathways of several sedimentary deposits relevant for oil exploration (Knudsen et al. 2005; Bernstein et al. 2008).

\section{Concluding remarks}

Earth science is becoming an increasingly more quantitative science with new insights, more often than not, derived from detailed measurements. The Titan Project represents an example where advanced geoscientific instrumentation has allowed us to design projects that were not previously feasible, thereby opening new scientific research areas (e.g. Bernstein et al. 2008; Keulen et al. 2008, 2009). It is, however, important to underline that this progress does not only include the scientific instruments and analyses themselves, it also represents a major challenge to store and analyse the huge quantities of data generated (Fig. 2). In this paper we have demonstrated that information technology plays a central role in facilitating the storage, retrieval and analysis of large geoscientific datasets.

Storing and distributing geological data are important aspects of national geological surveys, and it is important that GEUS follows in the footsteps of other national surveys in order to take full advantage of the new opportunities offered by the revolution of information technology. The Titan Project demonstrates that GEUS has the in-house expertise to develop technologies that address the full life cycle of geoscientific data from the raw instrument output, to store data securely in a database, analyse and reduce data, and finally distribute the data providing on-line and interactive access to the data. The know-how from the Titan Project is currently being applied to other similar projects at GEUS. Finally, we would like to point out that besides the scientific and societal rationale of Titan, it continues to be used by commercial partners. In fact, the application itself has almost exclusively been financed by industrial partners.

\section{References}

Bernstein, S., Frei, D., McLimans, R.K., Knudsen, C. \& Vasudev, V.N. 2008: Application of CCSEM to heavy mineral deposits: Source of high-Ti ilmenite sand deposits of South Kerala beaches, SW India. Journal of Geochemical Exploration 96, 25-42.

Keulen, N., Frei, D., Bernstein, S., Hutchison, M.T., Knudsen, C. \& Jensen, L. 2008: Fully automated analysis of grain chemistry, size and morphology by CCSEM: examples from cement production and diamond exploration. Geological Survey of Denmark and Greenland Bulletin 15, 93-96.

Keulen, N., Hutchison, M.T. \& Frei, D. 2009: Computer-controlled scanning electron microscopy: A fast and reliable tool for diamond prospecting. Journal of Geochemical Exploration 103, 1-5.

Knudsen, C., Frei, D., Rasmussen, T., Rasmussen, E.S. \& McLimans, R. 2005: New methods in provenance studies based on heavy minerals: an example from Miocene sands in Jylland, Denmark. Geological Survey of Denmark and Greenland Bulletin 7, 29-32.

Tulstrup, J. 2004: Environmental data and the Internet: openness and digital data management. Geological Survey of Denmark and Greenland Bulletin 4, 45-48. 